04

\title{
Моделирование плазмы короткодугового ксенонового разряда сверхвысокого давления
}

\author{
(c H.A. Тимофеевв, ${ }^{1}$ B.C. Сухомлинов, ${ }^{1}$ G. Zissis, ${ }^{2}$ И.Ю. Мухараева, ${ }^{1}$ Д.В. Михайлов, ${ }^{1}$ P. Dupuis ${ }^{3}$ \\ ${ }^{1}$ Санкт-Петербургский государственный университет, \\ 198504 Санкт-Петербург, Петергоф, Россия \\ ${ }^{2}$ LAPLACE, 118 route de Narbonne, \\ 31062 Toulouse, France \\ ${ }^{3}$ Kawantech SAS, \\ Toulouse, France \\ e-mail: niktimof@yandex.ru
}

Поступило в Редакцию 22 марта 2019 г.

В окончательной редакции 22 марта 2019 г.

Принято к публикации 10 апрлея 2019 г.

Исследован короткодуговой разряд высокого (сверхвысокого) давления в ксеноне, использующего катоды из торированного вольфрама. На основе ранее полученных экспериментальных данных, которые указывают на возможную эмиссию материала катода - тория, в разрядный промежуток, сформулирована система уравнений, позволяющая определить напряженность электрического поля, температуру плазмы, концентрации атомов тория и ионов тория и ксенона в плазме. Задача решена для модельного случая разряда между плоскими электродами. Полученные результаты свидетельствует о ключевой роли атомов тория в прикатодной области. Атомы тория определяют ионизационный баланс и другие электрокинетические свойства плазмы. Эмиссия атомов тория снижает температуру плазмы вблизи катода, которая оказывается заметно ниже температуры плазмы вблизи анода, что является новым результатом и находится в соответствии с экспериментальными данными. Также в хорошем согласии находятся другие электрокинетические характеристики плазмы, в частности, концентрация заряженных частиц и напряженность электрического поля.

Ключевые слова: короткодуговой ксеноновый разряд, высокое давление, торий, моделирование плазмы.

DOI: $10.21883 /$ JTF.2019.10.48171.103-19

\section{Введение}

Короткодуговые ксеноновые лампы высокого и сверхвысокого давления используются там, где требуется получить спектр излучения, максимально близкий к солнечному, и там, где источник излучения должен быть практически точечным. В этом отношении короткодуговые ксеноновые лампы не имеют аналогов и вряд ли могут быть замещены другими источниками оптического излучения.

Разряды высокого и сверхвысокого давления в инертных газах, на основе которых созданы в том числе и ксеноновые короткодуговые лампы, изучены достаточно хорошо $[1,2]$. Исследованы их оптические, спектроскопические, светотехнические и электрические характеристики. Это справедливо, как правило, для интегральных характеристик разряда: изучены полный световой поток, спектр излучения разряда как целого, светотехнические, электрические, энергетические характеристики. Существуют также немногочисленные исследования, в которых изучаются отдельные интересующие авторов аспекты работы таких источников света, направленные на решение конкретных научных, технических или других задач. Например, в работе [3] были экспериментально исследованы радиометрические характеристики инфракрасного излучения ксеноновых короткодуговых ламп мощностью $150 \mathrm{~W}$. Эти результаты были полезны для некоторых биомедицинских приложений и высокотемпературных реакторов, которые используют как видимое, так и инфракрасное излучение. В работе [4] изучалась эффективность рециркуляции света обратно в зону излучения лампы. Было показано, что такая рециркуляция может усилить излучение, полученное из плазмы, причем для определенных областей дуги яркость может быть увеличена до 70\%.

Однако ряд вопросов остается неисследованным. Прежде всего, это касается возможного присутствия атомов материала электродов в разряде и их влияния на свойства плазмы. Источники света на основе ксенонового разряда высокого и сверхвысокого давления имеют, как правило, торированные вольфрамовые катоды для уменьшения работы выхода и увеличения эмиссии электронов из материала катода. Сильный нагрев катода, вызванный большими плотностями тока, может привести к испарению атомов тория в разрядный промежуток. Торий имеет примерно вдвое более низкий потенциал ионизации $(6.3 \mathrm{eV})[5]$, чем ксенон $(12.13 \mathrm{eV})$, поэтому его присутствие в разрядном промежутке, безусловно, может сильно повлиять на характеристики плазмы. Сходный результат был получен в работе [6], где было 


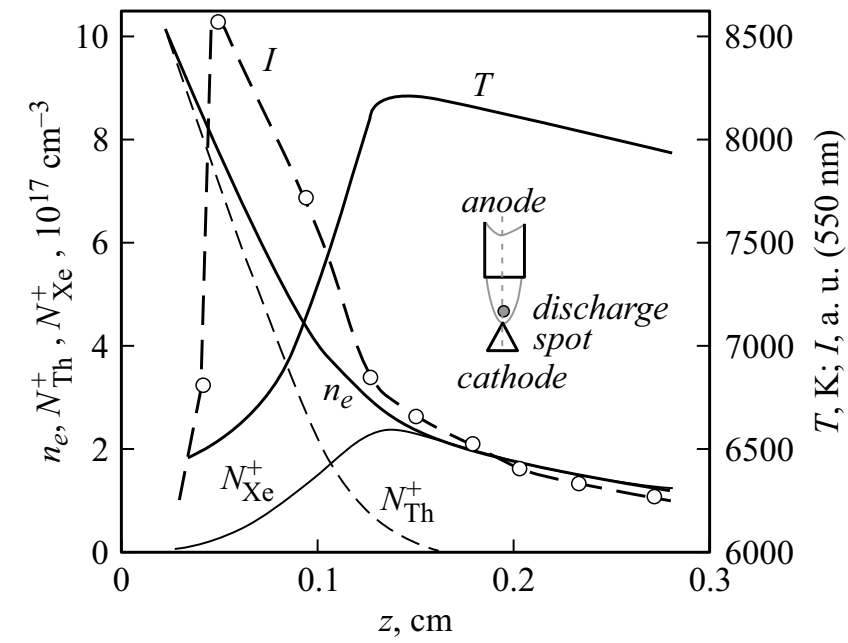

Рис. 1. Продольные распределения температуры плазмы $T$, концентраций электронов $n_{e}$, ионов тория $N_{\mathrm{Th}}^{+}$и ионов ксенона $N_{\text {Хе }}^{+}$; разряд в короткодуговой ксеноновой лампе сверхвысокого давления мощностью $250 \mathrm{~W}$ (давление ксенона в холодной лампе - $20 \mathrm{~atm})$; справа показана геометрия разряда.

показано, что присутствие в ксеноновом разряде атомов с низким потенциалом ионизации (в качестве добавки к атомам ксенона использовались молекулы галогенидов металлов, которые в условиях разряда разлагались на металл и галоген) существенно изменяло (улучшало) излучательные характеристики плазмы.

Проведенное ранее исследование спектра излучения вдоль оси ксенонового короткодугового разряда сверхвысокого давления [7,8] дало результаты, которые невозможно объяснить без учета эмиссии тория в разрядный промежуток: атомы тория, попадая в разрядный промежуток, уменьшали температуру плазмы, что существенно влияло на оптические характеристики разряда. Данные результаты были получены при исследовании короткодугового разряда стандартной ксеноновой лампы мощностью $250 \mathrm{~W}$. Разряд создавался между вольфрамовым торированным катодом, имеющим остроконечную форму, и массивным вольфрамовым анодом. Расстояние между электродами составляло $3 \mathrm{~mm}$, давление ксенона в холодной лампе - $20 \mathrm{~atm}$ (концентрация атомов ксенона на оси дуги $\sim 10^{20} \mathrm{~cm}^{-3}$ ), плотность тока вблизи катода $\sim 10^{3} \mathrm{~A} / \mathrm{cm}^{2}$. Вблизи катода возникала область (катодное пятно), имеющая максимальную интенсивность в видимой области; затем по мере приближения к аноду и расширения разряда, интенсивность излучения плазмы уменьшалась. На рис. 1 приведены продольные распределения концентрации заряженных частиц и температуры плазмы, полученные из спектральных измерений, а также интенсивность излучения плазмы вблизи $550 \mathrm{~nm}$. Схематично показана также геометрия разряда. Как видно из рис. 1, присутствие тория вблизи катода, действительно, уменьшает температуру плазмы $T$ и обеспечивает превалирование ионов тория $N_{\mathrm{Th}}^{+}$над ионами ксенона $N_{\mathrm{Xe}}^{+}$в этой части разряда.
При этом интенсивность излучения в видимой области спектра имеет максимум вблизи катода и не коррелирует с температурой плазмы, что невозможно объяснить в случае однородного по составу разряда (присутствие в плазме только атомов ксенона).

К сожалению, можно констатировать, что в силу определенных экспериментальных трудностей к настоящему времени нет надежных данных (экспериментальных, расчетных) о температуре катода короткодуговых ксеноновых ламп. Эти трудности связаны, по-видимому, с различной формой катода (цилиндрической, конической), разными добавками к материалу катода (вольфраму), величиной разрядного тока и другими условиями разряда (сортом газа и его давлением), что приводит к довольно большому разбросу в получаемой температуре катода: от $3000 \mathrm{~K}$ [9] (и даже ниже) до примерно $4000 \mathrm{~K}$ [10-15] (большинство этих данных получено для разряда в аргоне при атмосферном давлении). Наиболее ценными и интересными для нас являются результаты работы [16], в которой для ксенонового разряда высокого давления с катодом, допированным 2\% $\mathrm{ThO}_{2}$, была получена температура острия катода $3631 \mathrm{~K}$. Основываясь на температурной зависимости насыщенных паров тория над его поверхностью [17], можно заключить, что температура порядка $3600 \mathrm{~K}$ обеспечит концентрацию атомов тория вблизи катода $>10^{17} \mathrm{~cm}^{-3}$. Эта величина сравнима с ожидаемой концентрацией электронов [12,18], что в случае ионизации атомов тория должно сильно влиять на свойства плазмы. Работа катодов аргонового дугового разряда, для которого получен основной массив данных, сходна с работой катодов разряда в ксеноне, поэтому мы полагаем, что температура катода $\sim 3600 \mathrm{~K}$ и выше обеспечит значительное испарение атомов тория в прикатодную область разряда.

Целью настоящей работы является построение модели плазмы короткодугового разряда в ксеноне при высоком (сверхвысоком) давлении (слои вблизи электродов не включены в рассмотрение) с учетом влияния атомов тория на свойства плазмы.

\section{Основные уравнения}

Будем полагать, что плазма находится в условиях локального термодинамического равновесия $[1,2]$ и что в плазме присутствуют атомы тория, эмитированные торированным катодом. Геометрия разряда такова, что для описания пространственных распределений характеристик плазмы оказывается удобным использование эллиптических координат [19], где поверхности $\tau$ совпадают с эквипотенциальными поверхностями (с поверхностью электродов в том числе), а поверхности $\sigma-$ с силовыми линиями электрического поля. Такое представление позволяет сразу определить линии протекания электрического тока. Для нахождения параметров плазмы: температуры $T$, напряженности электрического поля $E$, концентрации атомов тория $N_{\mathrm{Th}}$, концентраций 
ионов тория $N_{\mathrm{Th}}^{+}$и ионов ксенона $N_{\text {Хе }}^{+}$, - были сформулированы и написаны пять уравнений: уравнения Саха для ионов тория и ионов ксенона, уравнение баланса мощности, уравнение тока через плазму и уравнение баланса числа атомов и ионов тория.

Уравнения Саха учитывали присутствие в плазме двух сортов ионов - ионов ксенона и ионов тория, сумма которых при выводе окончательных выражений заменяла концентрацию электронов $n_{e}=N_{\mathrm{Th}}^{+}+N_{\text {Хе }}^{+}$.

При формулировании уравнения баланса мощности учитывались нагрев плазмы в электрическом поле и потери энергии за счет излучения. Оценки показывают, что потери энергии за счет теплопроводности поперек разряда в силу его геометрии несущественны (поперечный диаметр кварцевой колбы составляет $\sim 2 \mathrm{~cm})$; также несущественны потоки тепла из плазмы на электроды: как показывают оценки, толщина слоя, в котором заметны потери мощности за счет потока на электроды, составляет $\sim 10^{-2} \mathrm{~cm}$. Это много меньше длины разрядного промежутка $0.3 \mathrm{~cm}$. Целью настоящей работы является модель плазмы без рассмотрения процессов в приэлектродных слоях, поэтому потери энергии на электродах не учитывались. Потери энергии за счет излучения складываются из рекомбинационно-тормозного излучения электронов на ионах ксенона и тория и линейчатого, главным образом, ИК-излучения атомов ксенона. Полное излучение в непрерывном спектре рассчитывалось с помощью выражения, приведенного в [20]; ИК-излучение атомов ксенона рассчитывалось суммированием наиболее ярких линий в диапазоне 800-1100 nm [21].

Соотношение для тока разряда представляло собой интеграл по поперечной координате $\sigma$. При этом для расчета подвижности электронов, как показываю оценки, в интересующих нас разрядных условиях достаточно учесть столкновения электронов с атомами ксенона и кулоновские столкновения заряженных частиц.

Для нахождения концентрации атомов тория были написаны уравнения для концентраций атомов и ионов тория в гидродинамическом приближении:

$$
\begin{aligned}
\frac{\partial N_{\mathrm{Th}}}{\partial t}= & \operatorname{div}\left(D_{\mathrm{Th}} \operatorname{grad} N_{\mathrm{Th}}\right)+\left(\frac{\delta N_{\mathrm{Th}}}{\delta t}\right)_{\mathrm{rec}}-\left(\frac{\delta N_{\mathrm{Th}}}{\delta t}\right)_{\text {ion }} \\
\frac{\partial N_{\mathrm{Th}}^{+}}{\partial t}= & \operatorname{div}\left(D_{\mathrm{Th}}^{+} \operatorname{grad} N_{\mathrm{Th}}^{+}\right) \\
& -\left(\frac{\delta N_{\mathrm{Th}}^{+}}{\delta t}\right)_{\mathrm{rec}}+\left(\frac{\delta N_{\mathrm{Th}}^{+}}{\delta t}\right)_{\mathrm{ion}}-\operatorname{div}\left(\overrightarrow{\mathrm{Th}^{+}}\right)
\end{aligned}
$$

Слагаемое $\left(\frac{\delta N_{\mathrm{Th}}}{\delta t}\right)_{\text {reс }}$ описывает появление атомов тория за счет рекомбинации ионов тория с электронами, слагаемое $\left(\frac{\delta N_{\text {Th }}}{\delta t}\right)_{\text {ion }}$ - уход атомов тория за счет ионизации, они равны соответственно уходу ионов тория за счет рекомбинации $\left(\frac{\delta N_{\mathrm{Th}}^{+}}{\delta t}\right)_{\text {rec }}$ и рождению ионов тория при ионизации $\left(\frac{\delta N_{\mathrm{Th}}^{+}}{\delta t}\right)_{\text {ion }},\left(\overrightarrow{J_{\mathrm{Th}^{+}}^{E}}\right)-$ поток ионов тория за счет

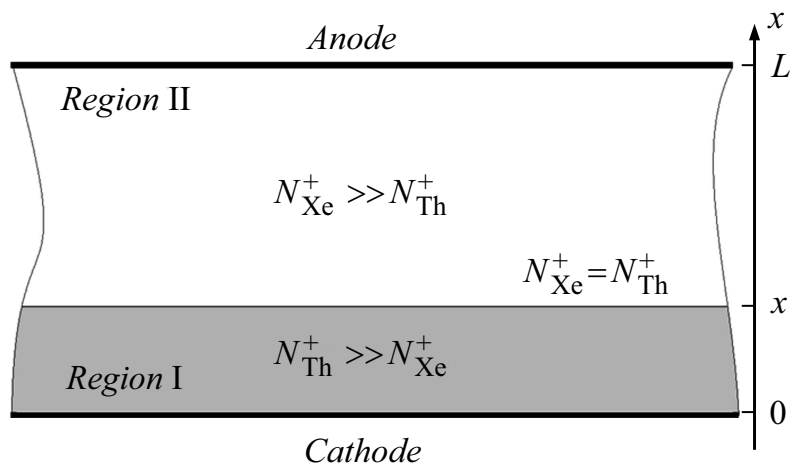

Рис. 2. Схематичное изображение одномерного разряда.

действия электрического поля, $D_{\mathrm{Th}}$ и $D_{\mathrm{Th}}^{+}-$коэффициенты диффузии атомов и ионов тория. Для разряда постоянного тока производные по времени равны нулю. Слагаемые, отвечающие за ионизацию и рекомбинацию, как показывают оценки, на 3-4 порядка больше слагаемых, отвечающих за диффузию и дрейф атомов и ионов тория. Это означает, что ионизация атомов тория уравновешивается объемной рекомбинацией, что ведет, очевидно, к уравнению Саха для ториевой компоненты плазмы. Складывая уравнения (1) и (2), легко получить уравнение для $N_{\mathrm{Th}}$, в котором не будет ионизационных и рекомбинационных членов:

$$
0=\operatorname{div}\left(D_{\mathrm{Th}} \operatorname{grad} N_{\mathrm{Th}}\right)+\operatorname{div}\left(D_{\mathrm{Th}}^{+} \operatorname{grad} N_{\mathrm{Th}}^{+}\right)-\operatorname{div}\left(j_{\mathrm{Th}^{+}}^{E}\right) .
$$

Таким образом, для определения пяти искомых характеристик плазмы - напряженности электрического поля $E$, температуры плазмы $T$, концентрации атомов тория $N_{\text {Th}}$, концентраций ионов ксенона $N_{\text {Хе }}^{+}$и ионов тория $N_{\mathrm{Th}}^{+}$(их сумма дает концентрацию электронов $\left.n_{e}\right)-$ имеется пять уравнений: два уравнения Саха, уравнение тока разряда, уравнение баланса мощности и уравнение баланса числа атомов тория (3).

\section{Упрощение задачи}

Задача, сформулированная в эллиптических координатах, с точки зрения получения количественных результатов, является достаточно сложной. В то же время наиболее важным, по нашему мнению, является вопрос о роли атомов тория в прикатодной области. Для выяснения этого вопроса в настоящей работе будет рассмотрена упрощенная с математической точки зрения задача в плоской геометрии с параметрами, близкими к параметрам короткодугового разряда высокого давления в ксеноне.

Положим, что разряд осуществляется между двумя плоскостями, линейные размеры которых много больше расстояния между ними. В этом случае задачу можно считать одномерной. На рис. 2 изображена геометрия такого разряда. Нижний электрод является катодом, 

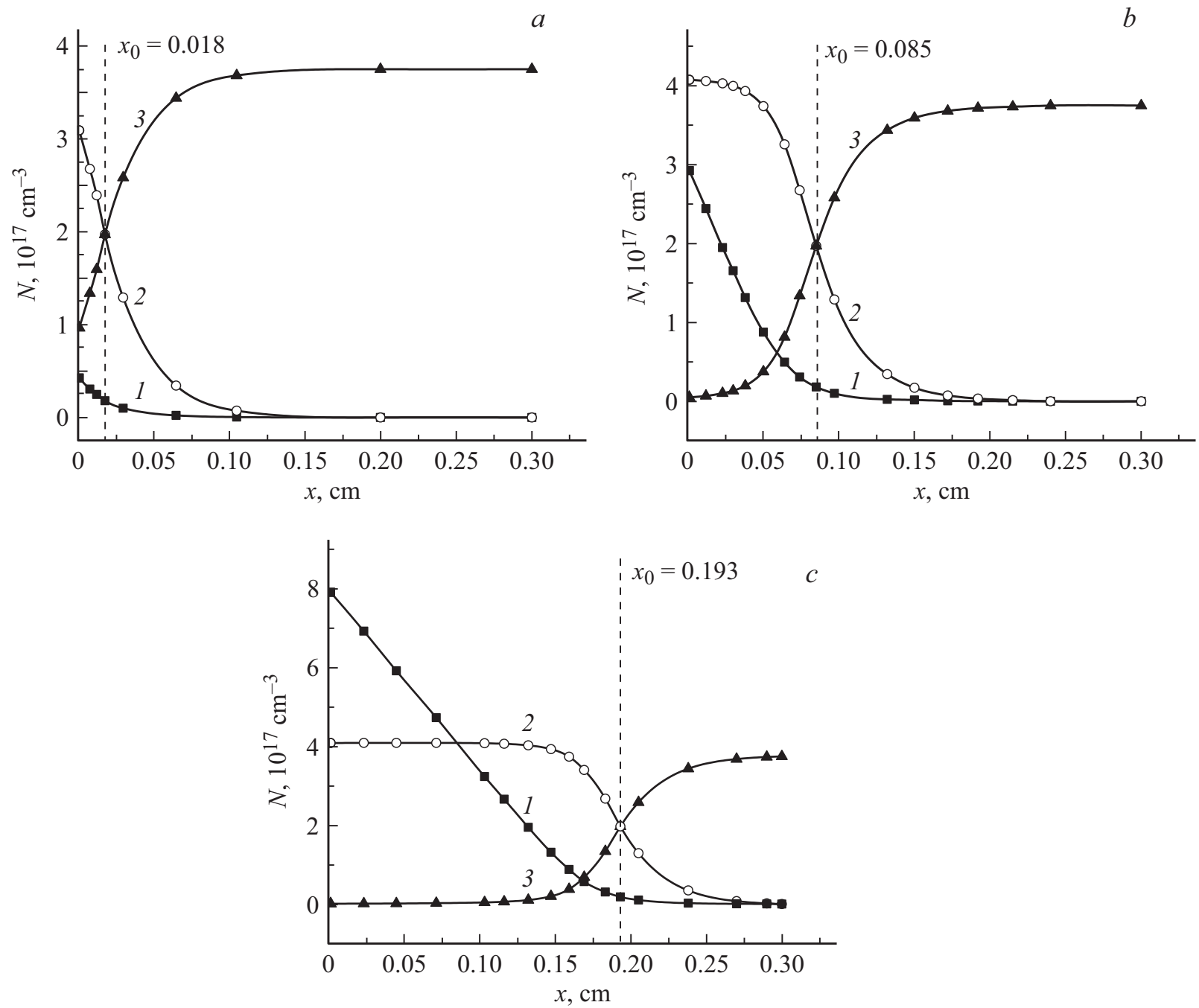

Рис. 3. Продольное распределение концентраций атомов тория (1), ионов тория (2) и ионов ксенона (3) при разной концентрации атомов тория на поверхности катода $N_{\mathrm{Th}}^{0} ; N_{\mathrm{Xe}}=3 \cdot 10^{19} \mathrm{~cm}^{-3} ; a-N_{\mathrm{Th}}^{0}=2 \cdot 10^{17} \mathrm{~cm}^{-3} ; b-N_{\mathrm{Th}}^{0}=5 \cdot 10^{17} \mathrm{~cm}^{-3}$; $c-N_{\mathrm{Th}}^{0}=10^{18} \mathrm{~cm}^{-3}$.

верхний - анодом, ось $x$ направлена от катода к аноду, при этом начало координат выбирается на поверхности катода (координата катода равна нулю), координата анода равна $L$. Разрядные условия будем считать близкими к условиям работы короткодуговой ксеноновой лампы сверхвысокого давления мощностью $250 \mathrm{~W}$ [1]: начальное давление ксенона (холодная лампа) $\sim 20$ atm, плотность тока $\sim 10^{3} \mathrm{~A} / \mathrm{cm}^{2}$, расстояние между электродами $0.3 \mathrm{~cm}$. Будем полагать, что температура поверхности острия катода $(\sim 3600 \mathrm{~K})$ обеспечивает концентрацию атомов тория $\sim\left(10^{17}-10^{18}\right) \mathrm{cm}^{-3}$. Эти значения вполне реальны, принимая во внимание данные работ [14-17].

Опираясь на результаты экспериментального исследования (рис. 1), положим, что область между электродами можно разбить на две части: область I, где концентрация ионов тория существенно превосходит концентрацию ионов ксенона, и область II, в которой концентрация ионов ксенона много больше концентрации ионов тория. Границей между этими двумя областями выберем по- верхность $x=x_{0}$, где концентрации ионов тория и ионов ксенона равны. Разбиение разрядного промежутка на две области I и II, предположение о превалировании разных сортов ионов в этих областях и использование баланса мощности и уравнений Саха для ионов тория и ксенона позволяют определить точку $x_{0}$, температуру плазмы в этой точке и получить аналитические решения $N_{\mathrm{Th}}(x)$ для областей I и II, а сшивка полученных функций $N_{\text {Th }}(x)$ и их производных - концентрацию атомов тория для всего разрядного промежутка. Совместное использование четырех оставшихся уравнений системы для всего разрядного промежутка позволяет найти $T(x)$, $E(x), N_{\mathrm{Th}}^{+}(x)$ и $N_{\mathrm{Xe}}^{+}(x)$.

\section{Результаты моделирования}

Определяющим в проводимом моделировании является распределение атомов и ионов тория в разрядном 
промежутке. Присутствие тория может привести к снижению температуры плазмы вблизи катода и сильному изменению других характеристик плазмы, прежде всего, оптических (излучательных). На рис. 3 приведены результаты расчета продольных распределений концентраций атомов тория $N_{\mathrm{Th}}$, ионов тория $N_{\mathrm{Th}}^{+}$и ионов ксенона $N_{\mathrm{Xe}}^{+}$при различной начальной концентрации атомов тория $N_{\mathrm{Th}}^{0}-$ концентрации атомов тория на поверхности катода: $N_{\mathrm{Th}}^{0}=2 \cdot 10^{17} \mathrm{~cm}^{-3}(a), 5 \cdot 10^{17} \mathrm{~cm}^{-3}(b)$, $10^{18} \mathrm{~cm}^{-3}(c)$. Из рис. 3 видно, что действительно при рассмотренных значениях $N_{\mathrm{Th}}^{0}$ вблизи катода наблюдается превалирование количества ионов тория над ионами ксенона в области I. При этом примечательно, что при двух меньших значениях $N_{\mathrm{Th}}^{0}$ концентрация ионов тория заметно выше концентрации атомов тория. Это означает, что атомы тория, попадая в разрядный промежуток, интенсивно ионизуются. Об этом свидетельствует также то, что, как видно из рисунков, аппроксимация концентрации атомов тория к точке $x=0$ дает меньшие значения, чем выбирались в качестве граничных условий. Например, для $N_{\mathrm{Th}}^{0}=2 \cdot 10^{17} \mathrm{~cm}^{-3}$ (рис. $3, a$ ) аппроксимация дает $0.6 \cdot 10^{17} \mathrm{~cm}^{-3}$, что примерно в три раза меньше концентрации на катоде.

Как и должно быть, концентрация ионов тория монотонно уменьшается, а концентрация ионов ксенона монотонно увеличивается с приближением к аноду. $\mathrm{B}$ точке $x=x_{0}$ эти концентрации равны. В точке $x_{0}$ концентрация атомов тория заметно меньше концентрации ионов тория. Это делает обоснованным выбранный нами способ решения, когда при нахождении $x_{0}$ мы полагали $N_{\mathrm{Th}}^{+} \gg N_{\mathrm{Th}}$. Вблизи анода атомов тория практически нет, и свойства плазмы определяются атомами ксенона. Это хорошо видно из рис. 3: концентрация $N_{\mathrm{Xe}}^{+}(L)$ вблизи

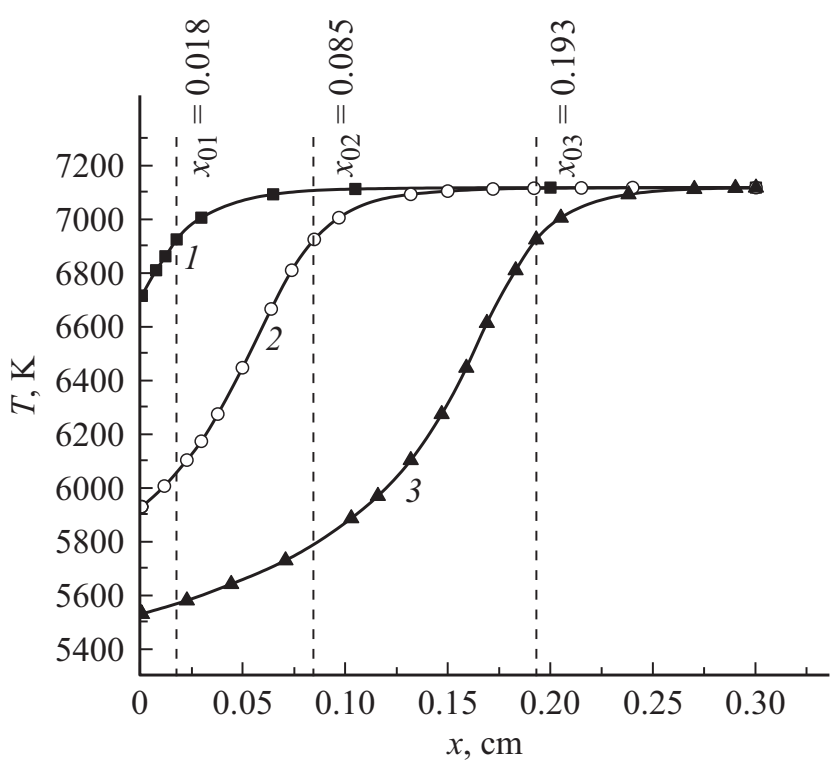

Рис. 4. Распределение температуры плазмы $T$ при разной концентрации атомов тория на поверхности катода; $\quad N_{\mathrm{Xe}}=3 \cdot 10^{19} \mathrm{~cm}^{-3} ; \quad 1-N_{\mathrm{Th}}^{0}=2 \cdot 10^{17} \mathrm{~cm}^{-3}$, $2-N_{\mathrm{Th}}^{0}=5 \cdot 10^{17} \mathrm{~cm}^{-3}, 3-N_{\mathrm{Th}}^{0}=10^{1} 8 \mathrm{~cm}^{-3}$.

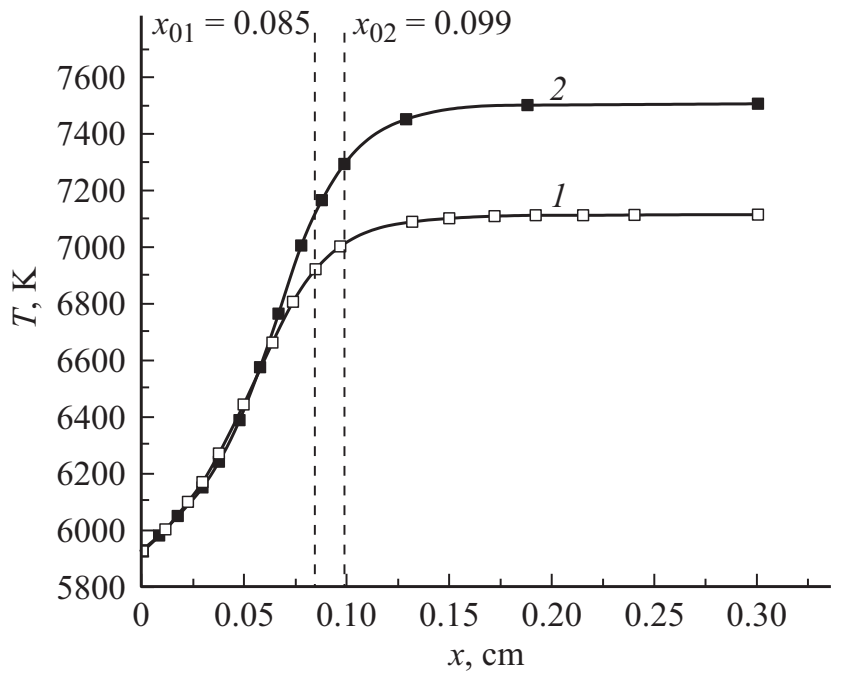

Рис. 5. Продольное распределение температуры плазмы $T$ при разной концентрации атомов ксенона; $N_{\mathrm{Th}}^{0}=5 \cdot 10^{17} \mathrm{~cm}^{-3}$; $1-N_{\mathrm{Xe}}=3 \cdot 10^{19} \mathrm{~cm}^{-3}, 2-N_{\mathrm{Xe}}=10^{19} \mathrm{~cm}^{-3}$.

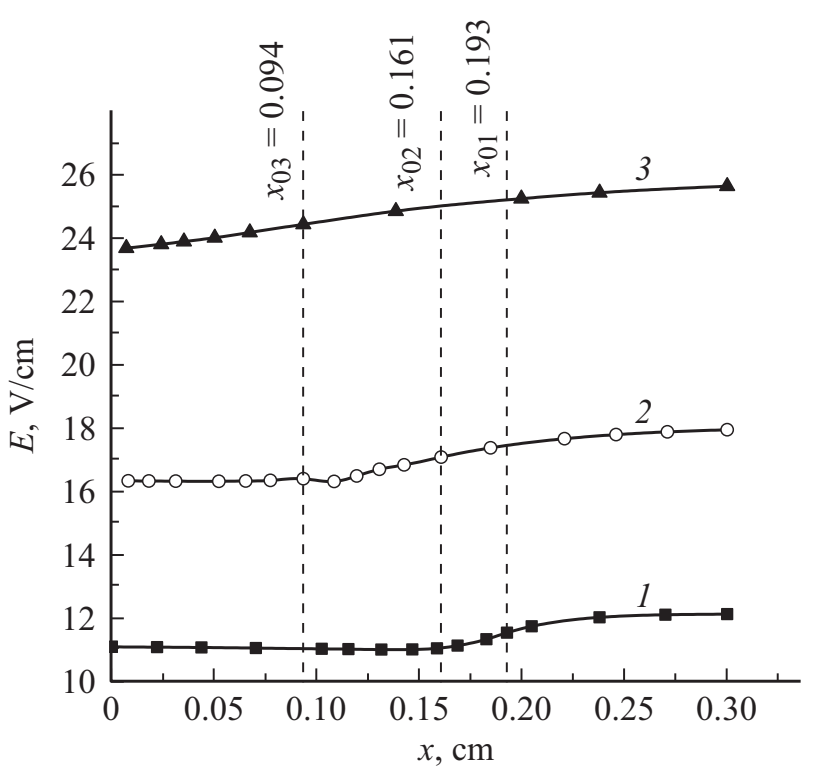

Рис. 6. Напряженность электрического поля $E$ при разной плотности тока $\left.j ; N_{\mathrm{Xe}}=3 \cdot 10^{19} \mathrm{~cm}^{-3}, N_{\mathrm{Th}}^{0}=10^{18} \mathrm{~cm}^{-3}\right) ; 1-$ $j=10^{3} \mathrm{~A} / \mathrm{cm}^{2}, 2-j=3.3 \cdot 10^{3} \mathrm{~A} / \mathrm{cm}^{2}, 3-j=10^{4} \mathrm{~A} / \mathrm{cm}^{2}$.

анода одинакова при разных $N_{\mathrm{Th}}^{0}$. Полученные значения концентрации электронов (сумма концентраций ионов тория и ксенона) по порядку величины $\sim 10^{17} \mathrm{~cm}^{-3}$ хорошо соответствуют данным работ $[12,18]$.

Рост начальной концентрации атомов тория $N_{\mathrm{Th}}^{0}$ приводит к удалению точки $x_{0}$ от катода, что вполне естественно. Это приводит также еще к одному интересному результату: концентрация ионов тория перестает превышать концентрацию атомов тория (pис. $3, c$ ).

На рис. 4 приведен расчет температуры плазмы $T(x)$ при различной начальной концентрации атомов тория на поверхности катода $N_{\mathrm{Th}}^{0}$. Присутствие тория в раз- 
ряде, как видно из рисунка, действительно уменьшает температуру плазмы вблизи катода, это уменьшение значительно и достигает $1500 \mathrm{~K}$ при изменении концентрации атомов тория вблизи катода в 5 раз. Полученный результат полностью подтверждает экспериментальные результаты, полученные из спектральных измерений $[7,8]$. Рост $N_{\mathrm{Th}}^{0}$ приводит к более сильному уменьшению температуры плазмы, что представляется естественным.

Уменьшение концентрации атомов ксенона приводит к слабому изменению температуры плазмы вблизи катода (все определяется торием) и к росту температуры в области, прилегающей к аноду. На рис. 5 приведены результаты расчета температуры плазмы при двух значениях концентрации атомов ксенона $-10^{19}$ и $3 \cdot 10^{19} \mathrm{~cm}^{-3}$. Полученный рост температуры вблизи анода связан с необходимостью поддержания требуемой концентрации заряженных частиц при уменышении концентрации ионизуемых атомов (ксенона) вблизи анода.

На рис. 6 представлен расчет напряженности электрического поля при различной плотности тока. Эти данные весьма интересны, потому что они фактически являются проверкой достоверности проведенного расчета. Напряженность электрического поля в короткодуговом разряде, очевидно, изменяется по длине разряда, тем не менее можно оценить усредненное значение. Оно составляет $\sim 20-50 \mathrm{~V} / \mathrm{cm}[1,22]$. В частности, для ксеноновой шаровой лампы при давлении ксенона $35 \mathrm{~atm}$ (холодная лампа) и разрядном токе $7.5 \mathrm{~A}$ была получена напряженность электрического поля $28 \mathrm{~V} / \mathrm{cm}$ [1]. В данной работе получено, что напряженность поля в силу геометрии решаемой задачи слабо зависит от продольной координаты и изменяется от 12 до $24 \mathrm{~V} / \mathrm{cm}$ с увеличением плотности тока на порядок величины. При плотности тока $10^{4} \mathrm{~A} / \mathrm{cm}^{2}$ напряженность поля равна $\sim 24 \mathrm{~V} / \mathrm{cm}$. Учитывая разную геометрию рассмотренной в данной работе задачи и геометрию реальной короткодуговой лампы это вполне удовлетворительное согласие, которое свидетельствует о правильности предлагаемой модели.

\section{Заключение}

В работе показано сильное влияние эмиссии материала катода (тория) в разрядный объем на характеристики плазмы: присутствие атомов тория заметно снижает температуру плазмы вблизи катода и полностью определяет ее электрокинетические характеристики. Хорошее качественное (в некоторых случаях - и количественное) согласие результатов расчета с данными эксперимента, несмотря на различие геометрии модельной задачи и геометрии реальной ксеноновой лампы, свидетельствует об адекватности учета определяющих свойства плазмы процессов и может служить основой создания модели плазмы короткодугового ксенонового разряда высокого (сверхвысокого) давления в реальной геометрии. Опи- санный в работе эффект может быть существенным для работы сильноточных дуговых разрядов, где также может быть сильным влияние эмиссии материала катода в разрядный промежуток.

Ксеноновые источники света (оптического излучения) высокого и сверхвысокого давления имеют, как правило, торированные катоды для уменьшения работы выхода электронов из материала катода. Торий является радиоактивным элементом, поэтому разрабатываются новые катоды, допированные другими элементами. В настоящее время эти исследования интенсивно ведутся, главным образом, с использованием редкоземельных элементов: $\mathrm{La}_{2} \mathrm{O}_{3}-\mathrm{W}, \mathrm{ZrO}_{2}-\mathrm{W}, \mathrm{CeO}_{2}-\mathrm{W}, \mathrm{Y}_{2} \mathrm{O}_{3}-$ [23], $\mathrm{Ce}-\mathrm{W}, \mathrm{La}-\mathrm{W}, \mathrm{Y}-\mathrm{W}$ [24]. Редкоземельные металлы имеют меньшие значения температуры плавления по отношению к вольфраму и потенциала ионизации по отношению к ксенону, поэтому можно полагать, что результаты данной работы будут применимы и для катодов, допированных другими элементами.

\section{Конфликт интересов}

Авторы заявляют, что у них нет конфликта интересов.

\section{Список литературы}

[1] Рохлин Г.Н. Разрядные источники света. М.: Энергоатомиздат, 1991. $720 \mathrm{c}$

[2] Lisnyak M., Cunha M., Bauchire J., Benolov M. // J. Appl. Phys. 2007. Vol. 50. N 31. P. 315203.

[3] Nakar D., Malul A., Feuermann D., Gordon J.M. // Appl. Opt. 2008. Vol. 47. N 2. P. 224-229. https://doi.org/10.1364/AO.47.000224

[4] Malul A., Nakar D., Feuermann D., Gordon J.M. // Opt. Express. 2007. Vol. 15. N 21. P. 14194-14201.

[5] Kohler S., Deissenberger R., Eberhardt K., Erdmann N., Herrmann G., Huber G., Kratz J.V., Nunnemann M., Passler G., Rao P.M., Riegel J., Trautmann N., Wendt K. // Spectrochim. Acta B. 1997. Vol. 52. P. 717-726. DOI: $10.1016 / \mathrm{S} 0584-8547(96) 01670-9$

[6] Neiger M., Hoppstock R., Kleiner B. US Patent 4937496. 1990.

[7] Тимофеев Н.А., Сухомлинов В.С., Зиссис Ж., Мухараева И.Ю., Дюпуа П. // Материалы Х Всероссийской конференции по физической электронике (ФЭ-2018). Махачкала, 2018. C. 19-23.

[8] Timofeev N., Sukhomlinov V., Zissis G., Mukharaeva I., Dupuis $P$. // IEEE. 2019. In press.

[9] Bergner A., Scharf F.H., Kuhn G., Ruhrmann C., Hoebing T., Awakowicz P., Mentel J. // Plasma Sources Sci. T. 2014. Vol. 23. N 5. P. 054005-054017. DOI: $10.1088 / 0963-0252 / 23 / 5 / 054005$

[10] Sillero J.L., Ortega D., Munoz-Serrano E., Casado E. // J. Phys. D: Appl. Phys. 2010. Vol. 43. N 18. P. 185204. https://hal.archives-ouvertes.fr/hal-00629948

[11] Reinelt J., Westermeier M., Ruhrmann C., Bergner A., Awakowicz P., Mentel J. // J. Phys. D: Appl. Phys. 2011. Vol. 44. N 9. 095204. doi.org/10.1088/0022-3727/44/9/095204 
[12] Zhu P., Lowke J.J., Morrow R. // J. Phys. D: Appl. Phys. 1992. Vol. 25. N 9. P. 1221-1230.

[13] Wendehtorr J., Wohlfahrt H., Simon G. // IEEE Conference Record - Abstracts. 1999 IEEE International Conference on Plasma Science. 26th IEEE International Conference (Cat. No 99CH36297). Monterrey, CA, USA. 2002. P. 240. DOI: 10.1109/PLASMA.1999.829559.

[14] Baeva M., Uhrlandt D., Benilov M.S., Cunha M.D. // Plasma Sources Sci. T. 2013. Vol. 22. P. 065017-065025. doi:10.1088/0963-0252/22/6/065017

[15] Baeva M. // Plasma Chem. Plasma P. 2017. Vol. 37. P. 341370. DOI: $10.1007 / \mathrm{s} 11090-017-9785-\mathrm{y}$.

[16] Minayeva O.B., Doughty D.A. // Proc of the $59^{\text {th }}$ GEC Conference. Columbus, OH, USA, 2006. P. RR2.00002.

[17] Stull D. American Institute of Physics Handbook / Ed. by D.E. Gray. NY.: McGraw Hill, 1972. 2200 p.

[18] Waymouth J.F. // IEEE. 1991. Vol. 19. N 6. P. 1003.

[19] Корн А.Г., Корн Т.М. Справочник по математике для научных работников и инженеров. М.: Наука, 1973. 832 с.

[20] Райзер Ю.П. Физика газового разряда. М.: Наука, 1987. $592 \mathrm{c}$.

[21] Kramida A., Ralchenko Yu., Reader J. NIST ASD Team. NIST Atomic Spectra Database (ver. 5.6.1), 2018. URL: https://physics.nist.gov/asd. National Institute of Standards and Technology, Gaithersburg, MD. DOI: https://doi.org/10.18434/T4W30F

[22] Ровинский P.E. // Светотехника. 1958. № 8. С. 5.

[23] Haidar J., Farmer A.J.D. // J. Phys. D: Appl. Phys. 1995. Vol. 28. P. 2089.

[24] Gu Z., Xi X., Yang J., Xu J. // Fuel. 2012. Vol. 95. P. 648. DOI: $10.1016 /$ j.fuel.2011.12.051 